\title{
Dietary Restriction Suppresses Steatosis-Associated Hepatic Tumorigenesis in Hepatitis C Virus Core Gene Transgenic Mice
}

\author{
Fangping Jia $^{a}$ Pan Diao $^{a}$ Xiaojing Wang ${ }^{a, b}$ Xiao $\mathrm{Hu}^{a, c}$ Takefumi Kimura ${ }^{d}$ \\ Makoto Nakamuta ${ }^{e}$ Ibuki Nakamura ${ }^{a}$ Saki Shirotoria ${ }^{a}$ Yoshiko Sato ${ }^{f}$ \\ Kyoji Moriyag Kazuhiko Koike ${ }^{h}$ Frank J. Gonzalez ${ }^{i}$ Jun Nakayama ${ }^{f}$ \\ Toshifumi Aoyama ${ }^{a}$ Naoki Tanakaa, j \\ a Department of Metabolic Regulation, Shinshu University School of Medicine, Matsumoto, \\ Japan; ${ }^{b}$ Department of Gastroenterology, Lishui Hospital, Zhejiang University School of \\ Medicine, Lishui, PR China; 'Department of Pathophysiology, Hebei Medical University, \\ Shijiazhuang, PR China; ${ }^{d}$ Department of Gastroenterology, Shinshu University School of \\ Medicine, Matsumoto, Japan; 'Department of Gastroenterology, Kyushu Medical Center, \\ Fukuoka, Japan; f Department of Molecular Pathology, Shinshu University School of Medicine, \\ Matsumoto, Japan; 9 Department of Infection Control and Prevention, The University of Tokyo, \\ Tokyo, Japan; h Department of Gastroenterology, The University of Tokyo, Tokyo, Japan; \\ 'Laboratory of Metabolism, National Cancer Institute, National Institutes of Health, Bethesda, \\ MD, USA; ${ }^{j}$ Research Center for Social Systems, Shinshu University, Matsumoto, Japan
}

\section{Keywords}

NF-kB · STAT3 $\cdot$ Cyclin D1 $\cdot$ p62/SQSTM1 $\cdot$ Senescence

\begin{abstract}
Background and Aims: Dietary restriction (DR) is a preventive strategy for obesity, metabolic syndrome, cardiovascular disease, and diabetes. Although an interconnection between obesity, metabolic syndrome, fatty liver, and hepatocellular carcinoma has been documented, the mechanism and impact of DR on steatosis-derived hepatocarcinogenesis are not fully understood. This study aimed to evaluate whether DR can prevent hepatic tumorigenesis. Methods: Male hepatitis C virus core gene transgenic ( $\mathrm{HCV}$ cpTg) mice that develop spontaneous age-dependent insulin resistance, hepatic steatosis, and ensuing liver tumor development without apparent hepatic fibrosis, were fed with either a control diet ad libitum (control group) or $70 \%$ of the same control diet (DR group) for 15 months, and liver phenotypes were investigated. Results: DR significantly reduced the number and volume of liver tumors. DR attenu-
\end{abstract}


ated hepatic oxidative and endoplasmic reticulum stress and markedly suppressed nuclear factor- $\mathrm{KB}$, signal transducer and activator of transcription 3 (STAT3) and STAT5, and phosphorylation of extracellular signal-regulated kinase, leading to downregulation of several prooncogenic mediators, such as cyclin D1. Serum insulin and insulin-like growth factor 1 levels, as well as hepatic expression of insulin receptor substrate 1/2, phosphatidylinositol-3 kinase, and serine/threonine-protein kinase AKT, were downregulated by DR. A transcriptome analysis revealed that STAT3 signaling and lipogenesis were the most suppressed hepatocarcinogenic pathways affected by DR. Additionally, DR stimulated autophagy and p62/sequestosome 1 degradation, enhanced phosphorylation of AMP-activated protein kinase $\alpha$, increased fibroblast growth factor 21 expression, and attenuated expression of senescence-associated secretory phenotypes. Conclusion: DR suppressed steatosis-associated hepatic tumorigenesis in HCVcpTg mice, mainly due to attenuation of pathways involved in inflammation, cellular stress, cell proliferation, insulin signaling, and senescence. These findings support the notion that persistent $30 \%$ reduction of daily food intake is beneficial for preventing steatosisassociated hepatocarcinogenesis caused by HCV core protein.

(C) 2020 The Author(s)

Published by S. Karger AG, Basel

\section{Introduction}

Epidemiological studies revealed that excess adiposity, decreased physical exercises, and unhealthy diets elevate the risk of cancers in various organs, especially in the liver [1, 2]. Hepatocellular carcinoma (HCC) is one of the common malignancies and leading causes of cancer-related death worldwide [3]. Persistent infection of hepatitis B virus or hepatitis C virus (HCV), ethanol consumption, and genetic metabolic disorders, such as hemochromatosis, Wilson's disease, glycogen storage disease, and citrin deficiency, are conventional risk factors for HCC $[4,5]$. Recently, worldwide increases in obesity and metabolic syndrome have raised the prevalence of HCC derived from non-alcoholic fatty liver disease (NAFLD) and nonalcoholic steatohepatitis (NASH) [1-8], indicating a close relationship between overnutrition and liver tumorigenesis.

Although hepatic steatosis may promote HCC development under persistent inflammation as a result of ethanol intake and hepatitis virus infection, NAFLD/NASH can singularly cause HCC from not only cirrhotic livers, but also livers with none-to-mild fibrosis [6, 7]. Accumulation of fatty acids (FA) and their intermediate metabolites in hepatocytes can enhance oxidative and endoplasmic reticulum (ER) stress, and lipid peroxidation, resulting in mitochondrial dysfunction, hepatocyte damage, and DNA mutations. With the progress of hepatocyte degeneration, Kupffer cells and hepatic stellate cells are activated and hepatic inflammation and fibrosis gradually progresses. The changes in hepatocyte integrity and microenvironment around hepatocytes initiate and promote malignant transformation of hepatocytes, eventually leading to HCC $[1,2,8,9]$. Therefore, correcting daily lifestyle and attenuating hepatosteatosis and adiposity are considered beneficial for preventing hepatic tumorigenesis associated with metabolic syndrome and NAFLD/NASH.

Dietary restriction (DR) is one of the promising strategies for reducing hepatic steatosis and systemic adiposity. Previous studies demonstrated that a chronic reduction of dietary energy intake by approximately 30\% significantly decreased adiposity and improved metabolic profiles in non-obese humans and rodents without accompanying malnutrition [10]. A positive correlation was noted between dietary intake and cumulative tumor incidence in mice [11]. Based on these findings, a hypothesis was developed that 30\% DR can attenuate steatosis-derived HCC occurrence. However, whether DR can actually prevent steatosisrelated hepatic tumorigenesis and its precise mechanism remains unclear. 
To address these issues, the current study used a HCV core gene transgenic (HCVcpTg) mouse line that spontaneously develops insulin resistance at age 2-3 months, hepatic steatosis at age 8-9 months, and hepatocellular tumors in approximately $30 \%$ of male mice between 16 and 18 months of age, without apparent hepatic inflammation and fibrosis, resembling the phenotype of NAFLD-associated hepatic tumorigenesis $[12,13]$. Therefore, the impact of persistent DR on steatosis-related hepatic tumorigenesis was investigated by conducting $30 \%$ reduction of food amount to HCVcpTg mice for 15 months.

\section{Materials and Methods}

\section{Mice and Treatment}

The generation of HCVcpTg mice on the C57BL/6J background has been described previously [12-15]. In this study, male 8- to 12-week-old HCVcpTg mice weighing 25-30 g were used. All mice were kept pathogen free according to institutional guidelines, and were under a 12:12-h light/dark cycle with free access to water. HCVcpTg mice were randomly divided into control $(n=17)$ or DR groups $(n=13)$ and fed either the AIN93G diet ad libitum or $70 \%$ of the average food amount of the control group for 15 months, respectively. The diet was purchased from Research Diets Inc. (New Brunswick, NJ, USA). Mice in the former group were also used as the controls in the other studies $[16,17]$. A $30 \%$ decrease in food was selected as the DR intervention for the following two reasons: (1) a 30\% food reduction protocol was reported to attenuate obesity and improve metabolic profiles without malnutrition in previous studies [10,11]; (2) this DR dose maintained the mouse body weight constant for the long term, preventing age-dependent body weight gain as determined by preliminary studies. Food consumption was determined every week in each cage. Body weight was measured weekly as well and food amounts adjusted to reproduce the absence of significant body weight gain in the DR group. After a 15-month treatment, the mice were killed by $\mathrm{CO}_{2}$ asphyxiation $6 \mathrm{~h}$ after food withdrawal. After the liver was removed, the liver surface was observed and the number and diameter of isolated tumors were determined. Subsequently, liver lobes were cut to 3-4 mm thickness using razors and the sections carefully evaluated to detect small tumors. Hepatic tumors were dissected from the surrounding non-tumorous tissue. Sera and non-tumor liver tissues were immediately frozen and stored at $-80^{\circ} \mathrm{C}$ until use. All animal experiments were conducted in accordance with animal study protocols outlined in the Guide for the Care and Use of Laboratory Animals from the National Academy of Sciences and all protocols were approved by the Shinshu University School of Medicine animal care and use committee.

\section{Biochemical Analysis}

Serum alanine aminotransferase (ALT), aspartate aminotransferase (AST), triglyceride (TG), total cholesterol (TC), non-esterified FA (NEFA), and glucose were measured with enzyme assay kits (Wako Pure Chemical Industries Ltd., Osaka, Japan). Serum concentrations of insulin, insulin-like growth factor 1 (IGF1), and adiponectin were determined using the mouse Insulin ELISA kit (TMB; AKRIN-011T, Shibayagi, Gunma, Japan), mouse/rat IGF-1/IGF1 Quantikine ELISA kit (MG100, R\&D Systems, Minneapolis, MN, USA), and mouse/rat adiponectin ELISA kit (Otsuka Pharmaceutical, Tokyo, Japan), respectively. Total liver lipids were extracted according to the hexane:isopropanol method [18] and measured using the identical kits (Wako Pure Chemical Industries Ltd). Hepatic hydroxyproline concentrations were measured using the QuickZyme Hydroxyproline Assay kit (QuickZyme BioSciences, Leiden, The Netherlands) as described elsewhere [16, 17].

Histological Analysis

Small pieces of liver tissue from each mouse were fixed in $10 \%$ buffered formalin and embedded in paraffin. Sections (3 $\mu \mathrm{m}$ thick) were stained with hematoxylin and eosin or the Azan-Mallory method [19, 20].

Expression of 4-hydroxy-nonenal (4-HNE), phosphorylated form of signal transducer and activator of transcription 3 (p-STAT3), and extracellular signal-regulated kinase (p-ERK) was analyzed by immunohistochemistry. Antigen retrieval was carried out by microwaving tissue sections in $10 \mathrm{~mm}$ Tris-HCl buffer (pH 8.0) containing $1 \mathrm{~mm}$ EDTA for $30 \mathrm{~min}$ for immunostaining. The sections were incubated $1 \mathrm{~h}$ with an anti4-HNE antibody (No. MHN-020P, JaICA, Shizuoka, Japan, 1:5 dilution), anti-p-STAT3 antibody (No. 9145, Cell Signaling Technology, Danvers, MA, USA, 1:100 dilution), and anti-p-ERK antibody (No. 4370, Cell Signaling

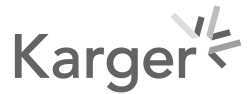


Technology, 1:500 dilution), respectively. For secondary antibodies, Histofine mousestain for 4-HNE and Histofine Simple Stain MAX-PO ${ }^{\circledR}$ for others, both purchased from Nichirei (Tokyo, Japan), were used. For immunodetection, peroxidase activity was visualized using diaminobenzidine-hydrogen peroxide solution. Specific staining was not detected in control experiments omitting the primary antibody. Pathological diagnosis was performed by J.N., a certified pathologist by the Japanese Society of Pathology, and N.T. in an independent and blinded manner.

\section{Quantitative Polymerase Chain Reaction Analysis}

Total liver RNA was extracted using NucleoSpin RNA Plus Kit (Macherey-Nagel GmbH \& Co. KG, Düren, Germany) and cDNA was reverse-transcribed with ReverTra Ace ${ }^{\circledR}$ quantitative polymerase chain reaction (qPCR) RT Master Mix (Toyobo, Osaka, Japan). The levels of mRNA were quantified using an SYBR qPCR kit (Toyobo) and Applied Biosystems StepOne Plus Real-time PCR system (Thermo Fisher Scientific Inc., Waltham, MA, USA) [16-22]. One microliter of cDNA aliquots was added to each well. The mRNA levels were normalized to those of $18 \mathrm{~S}$ ribosomal RNA and expressed as fold changes relative to HCVcpTg mice fed the control diet ad libitum. The primer pairs used for qPCR analysis are listed in online supplementary Table 1 (for all online suppl. material, see www.karger.com/doi/10.1159/000508308).

\section{Microarray Analysis}

Total RNA was extracted using TRIzol reagent (Thermo Fisher Scientific Inc.) and was purified with the Qiagen RNeasy Mini Kit (Qiagen, Valencia, CA, USA). RNA quantity and quality were detected using a Nanodrop One spectrophotometer (Thermo Fisher Scientific Inc.) and an Agilent Bioanalyzer (Agilent Technologies, Santa Clara, CA, USA). Total RNA was amplified and labeled with Cyanine 3 (Cy3) using an Agilent Low Input Quick Amp Labeling Kit, one-color (Agilent Technologies) following the manufacturer's instructions. Briefly, total RNA was reverse transcribed to double-strand cDNA with a poly dT-T7 promoter primer. Primer, template RNA, and quality-control transcripts of known concentration and quality were first denatured at $65^{\circ} \mathrm{C}$ for $10 \mathrm{~min}$ and incubated for $2 \mathrm{~h}$ at $40^{\circ} \mathrm{C}$ with $5 \times$ first strand buffer, $0.1 \mathrm{M} \mathrm{DTT}, 10 \mathrm{~mm} \mathrm{dNTP}$ mix, and AffinityScript RNase Block Mix. The AffinityScript enzyme was inactivated at $70^{\circ} \mathrm{C}$ for $15 \mathrm{~min}$. The cDNA products were then used as templates for in vitro transcription to generate fluorescent cRNA. In brief, cDNA products were mixed with a transcription master mix in the presence of T7 RNA polymerase and Cy3 labeled$\mathrm{CTP}$ and incubated at $40^{\circ} \mathrm{C}$ for $2 \mathrm{~h}$. Labeled cRNAs were purified using Qiagen's RNeasy mini spin columns and eluted in $30 \mu \mathrm{L}$ of nuclease-free water. After amplification and labeling, Nanodrop One spectrophotometer (Thermo Fisher Scientific Inc.) and Agilent Bioanalyzer were used to measure cRNA quantity and cyanine incorporation. For each hybridization, $1.65 \mu \mathrm{g}$ of Cy3 labeled cRNA were fragmented, and hybridized at $65^{\circ} \mathrm{C}$ for $17 \mathrm{~h}$ to an Agilent SurePrint G3 Mouse GE 4x44K v2 Microarray (design ID: 026655). After washing, microarrays were scanned using an Agilent SureScan Microarray Scanner System (G4900DA). Agilent feature extraction software version 11.5.1.1 was used to measure the intensity values of each scanned feature and the background subtraction was performed. We only used features which were flagged as no errors (detected flags) and excluded features which were not positive, not significant, not uniform, not above background, saturated, and population outliers (not detected and compromised flags). Normalization was performed using Agilent GeneSpring software version 14.9.1 (per chip: normalization to 75 percentile shift).

\section{Immunoblot Analysis}

Whole liver lysate was extracted as described previously and hepatic nuclear fractions were isolated with NE-PER Nuclear and Cytoplasmic Extraction Reagents (Thermo Fisher Scientific Inc.). The protein concentration was measured using a BCA Protein Assay Kit (Pierce Biotechnology, Rockford, IL, USA). Whole liver homogenates (25-50 $\mu \mathrm{g}$ protein in each lane) were subjected to sodium dodecyl sulfate-polyacrylamide gel electrophoresis (SDS-PAGE) at a polyacrylamide gel concentration of 7.5-15\% [14-24]. Nuclear fractions (21 $\mu$ g of protein in each lane) were separated using 10\% SDS-PAGE for assessing nuclear factor-kappa B (NF- $\kappa \mathrm{B})$ p65 component and peroxisome proliferator-activated receptor- $\alpha$ (PPAR $\alpha$ ). After electrophoresis, proteins were transferred to nitrocellulose or polyvinylidene difluoride membranes (Amersham Hybond-P, GE Healthcare, Little Chalfont, UK). The membranes were blocked with 1-10\% bovine serum albumin or 3-10\% non-fat dry milk in Tris-buffered saline for $1 \mathrm{~h}$ at room temperature and incubated with the primary antibodies listed in online supplementary Table 2 overnight, followed by alkaline phosphatase-conjugated secondary antibodies (No. 93785, Jackson ImmunoResearch Laboratories, West Grove, PA, USA, 1:3000 dilution), and performed with 1-step NBT/BCIP (nitro-blue tetrazolium chloride/5-bromo-4-chloro-3'-indolyphosphate p-toluidine salt) substrate (Pierce Biotechnology) or 
Jia et al.: Dietary Restriction Suppresses Steatosis-Derived Hepatocarcinogenesis

horseradish peroxidase-conjugated secondary antibodies (No. 115-035-003, Jackson ImmunoResearch Laboratories, 1:4000 dilution) and performed with Amersham ECL Prime Western Blotting Detection Reagents RPN2232 (GE Healthcare). We co-electrophoresed molecular weight standards (Bio-Rad Laboratories, Hercules, CA, USA) to determine the correct position of immunostained bands. The band intensity was measured densitometrically and then normalized to that of $\beta$-actin (ACTB) or histone H1 and normalized to that of mice fed an AIN93G diet ad libitum ultimately. Immunoblot analysis was performed at least twice for each target protein.

Statistical Analysis

Values are presented as the mean \pm standard error of the mean (SEM). The quantitative and qualitative data analysis was performed with the two-tailed Student $t$ test and $\chi^{2}$ test, respectively, using SPSS statistics version 22 (IBM, Armonk, NY, USA). A $p$ value $<0.05$ was considered statistically significant.

\section{Results}

\section{DR Suppresses Hepatic Tumorigenesis in HCVcpTg Mice}

A 30\% DR 15-month study was conducted using male HCVcpTg mice (Fig. 1a). In the DR group, the number and volume of liver tumors, as well as body weight, were reduced compared with the control group (Fig. 1b, c). Serum glucose, AST, ALT, TC, TG, and NEFA were similar between the groups (Fig. 1d; online suppl. Table 3). Liver histology revealed attenuation of macrovesicular steatosis in the DR group (Fig. 1e), while the decreases in hepatic TC/TG contents did not reach statistical significance (online suppl. Table 3), presumably due to heterogeneous fat accumulation in the liver.

\section{DR Suppresses Hepatic NF- $\kappa B$ Activity in HCVcpTg Mice}

Since inflammatory response plays an important role in the tumorigenic process at various stages, including initiation, promotion, malignant conversion, invasion, and metastasis [25], the expression of key inflammatory mediators was investigated. The levels of cluster of differentiation 68 (Cd68) and myeloperoxidase (Mpo) mRNAs encoding conventional markers of macrophages and neutrophils, respectively, were significantly reduced in the DR group (Fig. 2a). The same tendency was observed in pro-inflammatory cytokines released from M1 macrophage, such as tumor necrosis factor $\alpha$ (Tnf), interleukin (IL)-1 $\beta$ $(I l 1 b)$, colony-stimulating factor 1 (Csf1), and C-C motif chemokine ligand 2 (CCL2, also known as monocyte chemoattractant protein-1, encoded by Ccl2; Fig. 2a), while there was no significant difference between the two groups in the levels of arginase 1 (Arg1) mRNA (Fig. 2a), an indicator of M2 polarization. The levels of suppressor of cytokine signaling 3 (Socs3) mRNA were reduced (Fig. 2a). The other markers of inflammation, galectin-3 (Lgals3) and its binding protein (Lgals3bp) and secreted phosphoprotein 1 (osteopontin, encoded by Spp1) mRNAs were also reduced (Fig. 2a). These pro-inflammatory mediators are induced by activation of NF- $\kappa \mathrm{B}$ mainly through nuclear translocation of NF- $\kappa \mathrm{B}$ complex. Immunoblot analysis of nuclear fractions confirmed that 30\% DR significantly reduced nuclear p65 contents (Fig. 2b).

Inflammasome and Toll-like receptor (TLR) signaling are known to activate NF- $\kappa \mathrm{B}$ [25]. The mRNA levels of inflammasome-related genes, including PYD and CARD domain containing (ASC, encoded by Pycard) and pannexin 1 (Panx1) were significantly reduced in the DR group (Fig. 2c). Proteolytically cleaved caspase 1 (CASP1) is a definitive indicator of inflammasome activation. Indeed, immunoblot analysis uncovered reduced cleaved CASP1 in the DR group (Fig. 2d). On the contrary, the mRNA levels from TLR-related genes are similar between the groups (Fig. 2e). These results indicate that DR inhibits NF- $\kappa B$ activity mainly through suppressing the inflammasome pathway. 


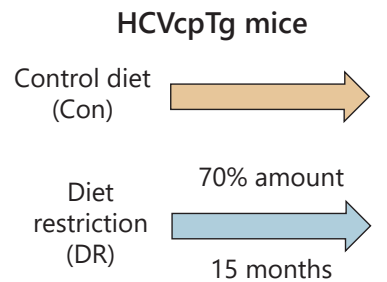

a
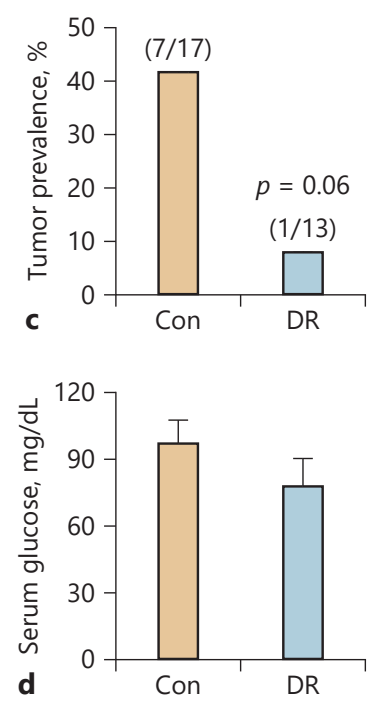
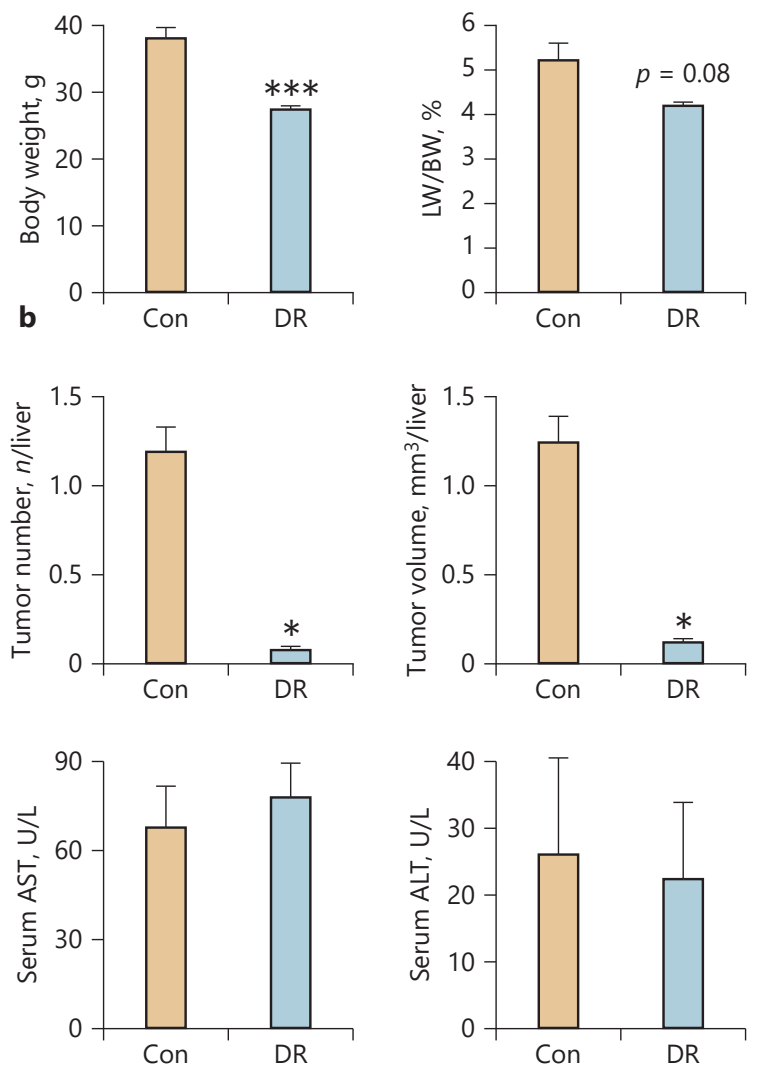
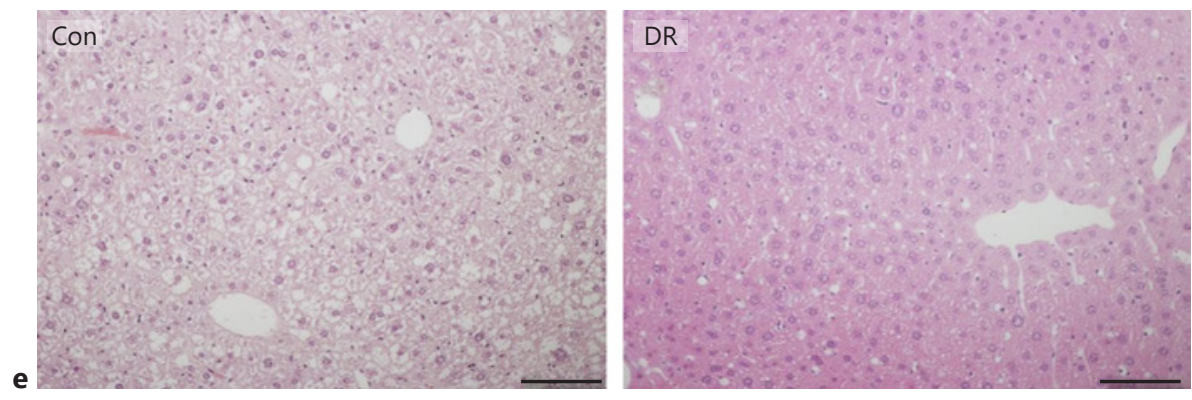

Fig. 1. 30\% DR for 15 months suppresses hepatic tumorigenesis in HCVcpTg mice. a Protocol of this study. b Body weight and liver-to-body weight ratio (LW/BW). c Liver tumor prevalence, number, and volumes. d Serum glucose, AST, and ALT levels. e Representative photomicrographs of hematoxylin and eosin-stained liver sections. Scale bar, $100 \mu \mathrm{m}$. Macrovesicular steatosis was attenuated in HCVcpTg after DR. Data are expressed as the mean \pm SEM. $* p<0.05$ and ${ }^{* * *} p<0.001$ between regularly fed control (Con) versus $30 \%$ reduction in diet (DR) groups.

\section{DR Reduces Hepatic Oxidative and ER Stress in HCVcpTg Mice}

Attenuated oxidative and ER stress downregulate NF- $\mathrm{KB}$ signaling and reduce the production of highly toxic metabolites, e.g., lipid peroxides and ensuing DNA damage, contributing to suppression of hepatic tumorigenesis [26, 27]. Hepatic 4-HNE, a major lipid peroxidation aldehyde, was markedly decreased in the DR group (Fig. 3a). Immunostaining for 4-HNE showed that it was accumulated in the cytoplasm of hepatocytes in control HCVcpTg 

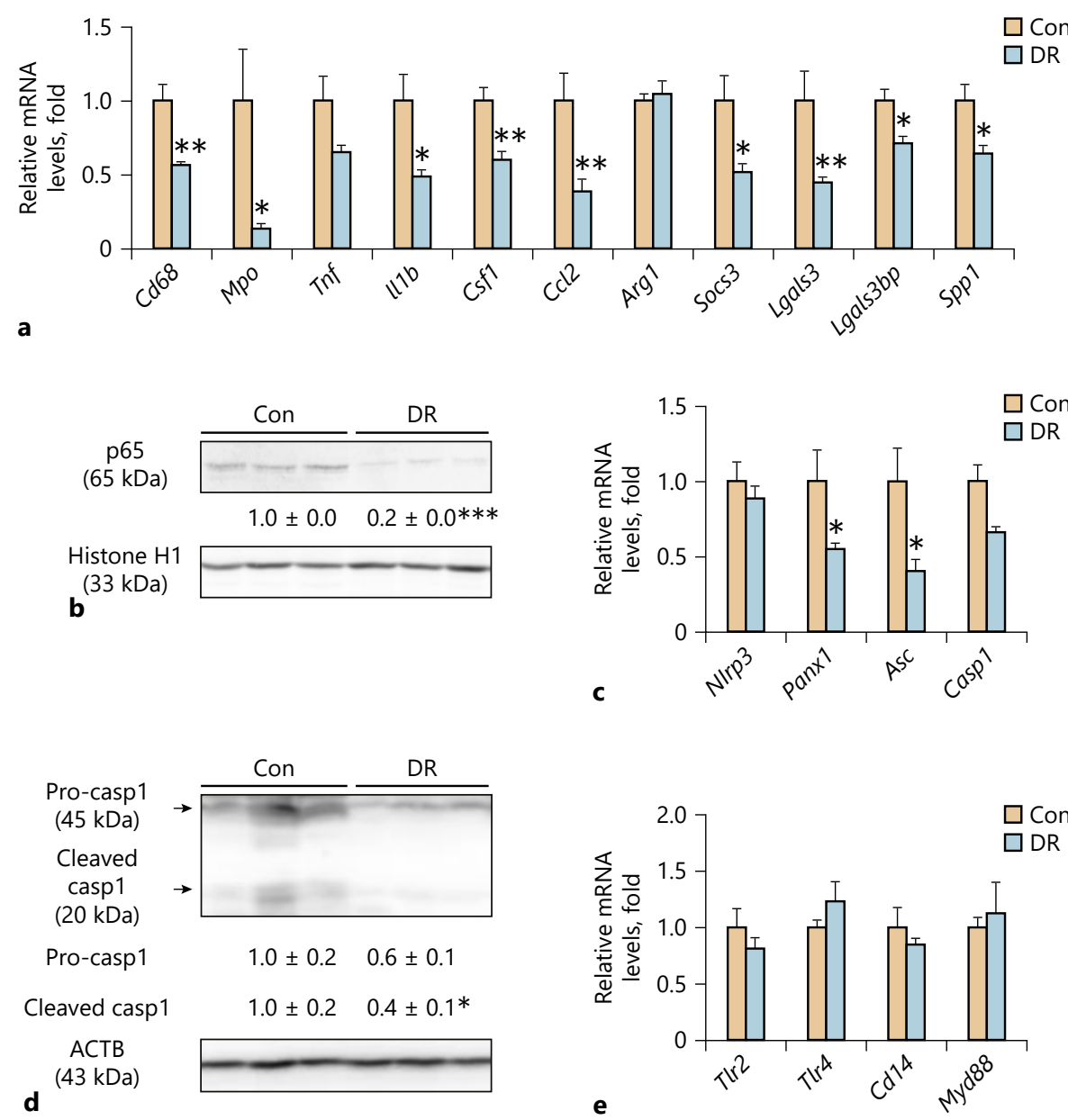

Fig. 2. 30\% DR reduces the expression of pro-inflammatory mediators through suppressing hepatic inflammasome and NF- $\kappa B$ in HCV cpTg mice. a Hepatic mRNA levels of genes encoding inflammatory cell indicators (Cd68 and Mpo), M1 cytokines (Tnf, Il1b, Csf1, and Ccl2), M2 cytokines (Arg1), STAT3 signaling suppressor (Socs3), and the other pro-inflammatory mediators (Lgals3, Lgals3bp, and Spp1) were quantified by qPCR, normalized to $18 \mathrm{~S}$ ribosomal RNA, and expressed as values relative to regularly fed control HCVcpTg mice. b, d Immunoblot analysis of NF- $\kappa$ B p65 (b) and caspase 1 (d). Nuclear fractions (21 $\mu \mathrm{g}$ protein/lane) and whole liver lysates (45 $\mu \mathrm{g}$ protein/lane) were separated using 10\% SDS-PAGE and the bands of histone H1 and $\beta$-actin (ACTB) were used as a loading control, respectively. The caspase 1 antibody detected pro-caspase 1 (45 kDa) and cleaved (activated) caspase $1(20 \mathrm{kDa})$. Band intensities were measured densitometrically, normalized to the loading control, and expressed as values relative to those of regularly fed control HCVcpTg mice. Results were obtained from at least two independent immunoblot experiments and representative blots are shown. c, e Hepatic mRNA levels of genes involved in inflammasomes (Nlrp3, Panx1, Asc, and Casp1; c) and TLRs (Tlr2, Tlr4, Cd14, and Myd88; e) were quantified by qPCR, normalized to 18S ribosomal RNA, and expressed as values relative to regularly fed control HCVcpTg mice. Data are expressed as the mean \pm SEM. ${ }^{*} p<0.05,{ }^{* *} p<0.01$, and ${ }^{* * *} p<0.001$ between regularly fed control (Con) versus $30 \%$ reduction in $\operatorname{diet}(\mathrm{DR})$ groups.

mice, while 4-HNE-positive hepatocytes were not observed in DR-treated mice (Fig. 3b). The mRNA levels of genes encoding reactive oxygen species-generating enzymes, such as cytochrome b-245 beta chain (CYBB) and neutrophil cytosolic factor 1 (NCF1), were decreased by DR (Fig. 3c). The expression of mRNAs encoding superoxide dismutase 1 (SOD1), a major 


\begin{tabular}{|c|c|c|}
\hline \multirow[b]{2}{*}{ Liver Cancer } & \multicolumn{2}{|l|}{ Liver Cancer 2020;9:529-548 } \\
\hline & DOI: $10.1159 / 000508308$ & $\begin{array}{l}\text { (c) } 2020 \text { The Author(s). Published by S. Karger AG, Basel } \\
\text { www.karger.com/lic }\end{array}$ \\
\hline
\end{tabular}

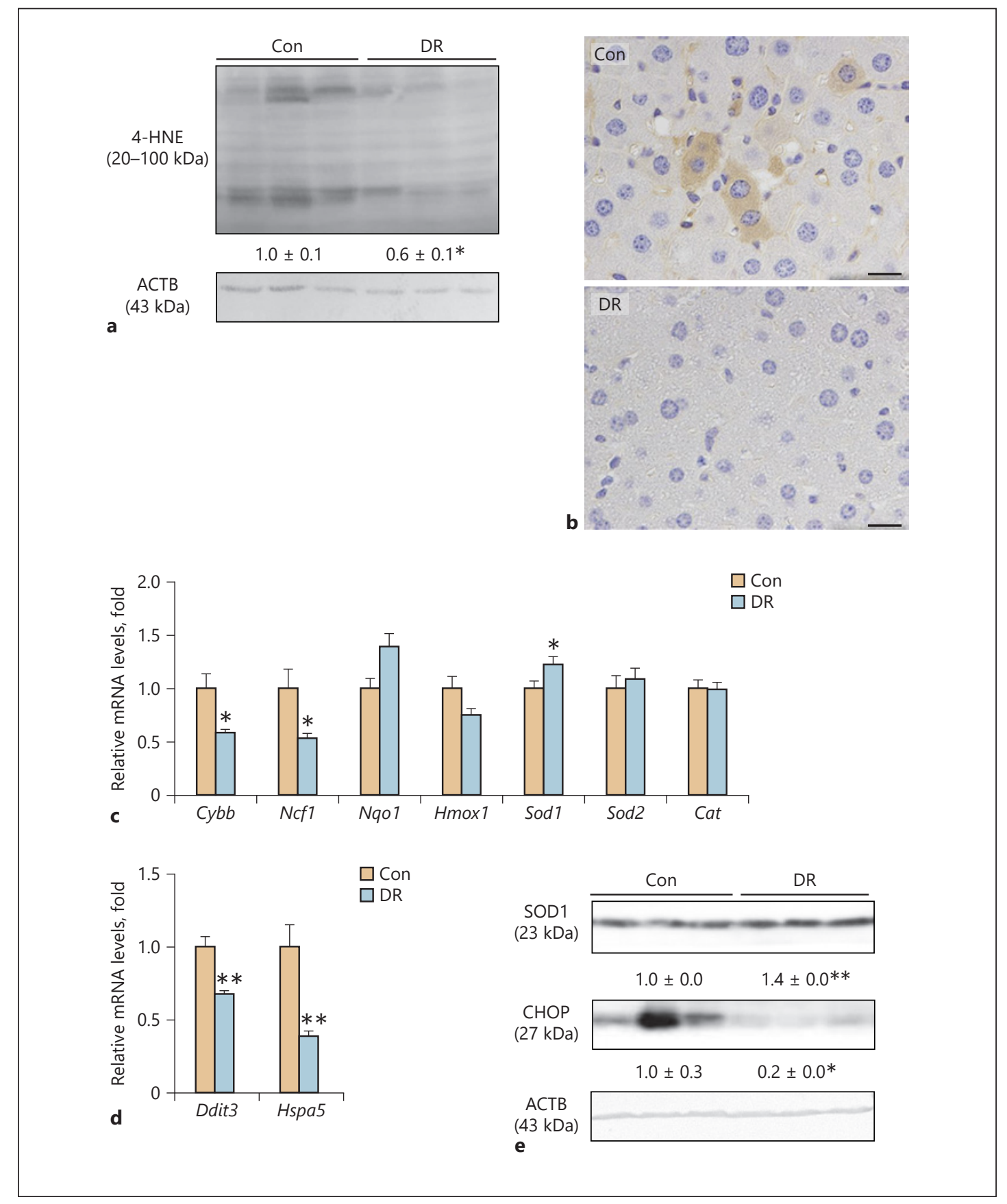

Fig. 3. 30\% DR reduces hepatic oxidative and ER stress in HCVcpTg mice. a, e Immunoblot analysis of 4-HNE (a), SOD1 and CHOP (e). Whole liver homogenates ( $45 \mu \mathrm{g}$ protein/lane) were separated using 10\% SDS-PAGE and the $\beta$-actin (ACTB) band was used as a loading control. Band intensities were measured densitometrically, normalized to the loading control, and expressed as values relative to those of regularly fed control HCVcpTg mice. Results were obtained from at least two independent immunoblot experiments and representative blots are shown. b Immunohistochemical staining for 4-HNE. 4-HNE was positive for cytoplasms of hepatocytes in the control HCVcpTg mice, but 4-HNE-positive hepatocytes were not observed in the DR group. Scale bar, $40 \mu \mathrm{m}$. c, d Hepatic mRNA levels of genes involved in oxidative stress generation (Cybb and Ncf1) and elimination (Nqo1, Hmox1, Sod1, Sod2, and Cat; c) and ER stress (Ddit3 and Hspa5; d) were quantified by qPCR, normalized to $18 \mathrm{~S}$ ribosomal RNA, and expressed as values relative to regularly fed control HCVcpTg mice. Data are expressed as the mean \pm SEM. $* p<0.05$ and ${ }^{* *} p<0.01$ between regularly fed control (Con) versus $30 \%$ reduction in diet (DR) groups.

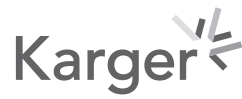




\begin{tabular}{|c|c|c|}
\hline \multirow[b]{2}{*}{ Liver Cancer } & \multicolumn{2}{|l|}{ Liver Cancer 2020;9:529-548 } \\
\hline & DOI: $10.1159 / 000508308$ & $\begin{array}{l}\text { O) } 2020 \text { The Author(s). Published by S. Karger AG, Basel } \\
\text { www.karger.com/lic }\end{array}$ \\
\hline
\end{tabular}

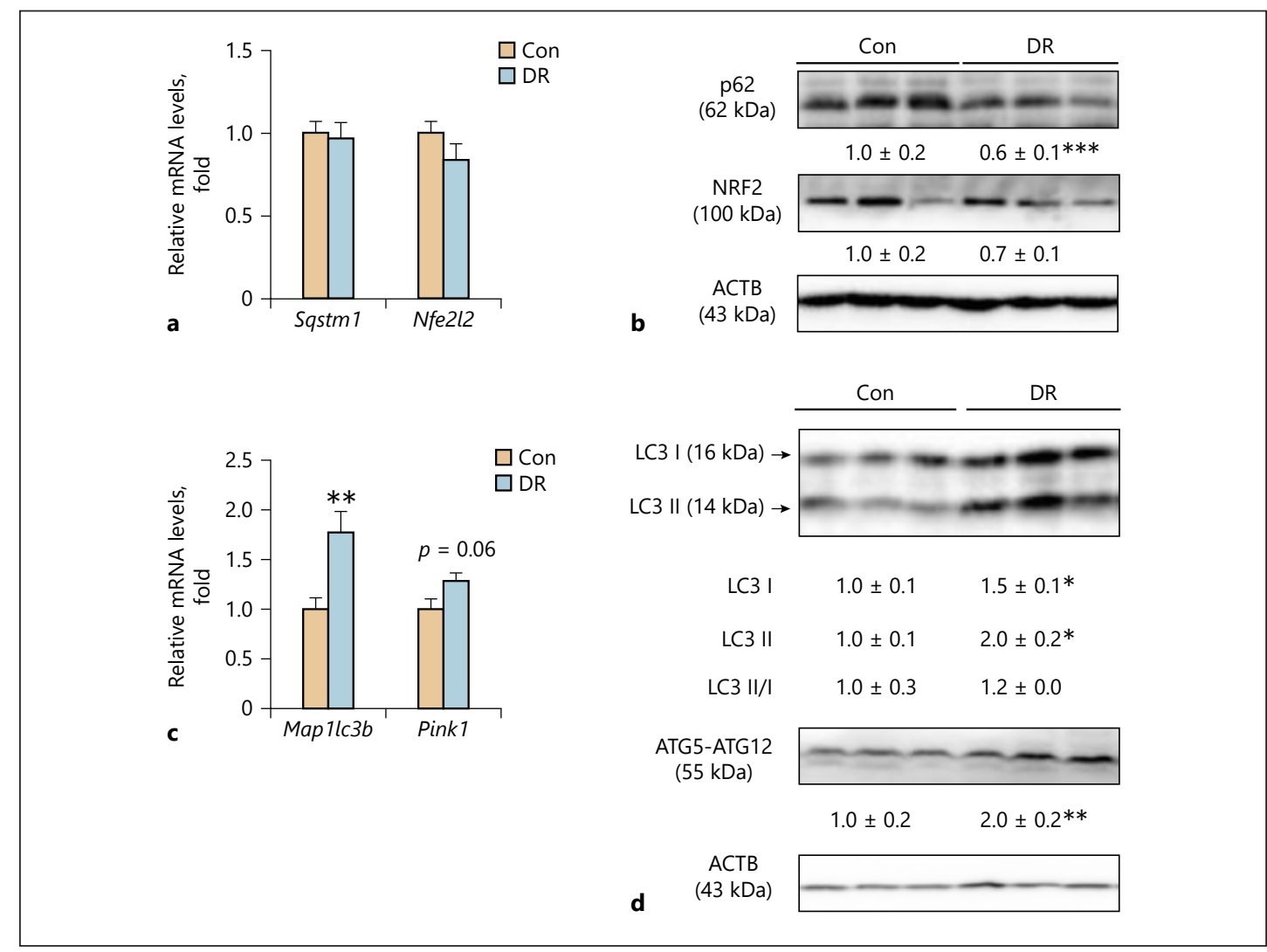

Fig. 4. $30 \%$ DR enhances autophagy and p62/sequestosome 1 degradation in HCVcpTg mice. a, c Hepatic mRNA levels of p62 (Sqstm1), NRF2 (Nfe2l2; a), and autophagy-associated genes (c). The same samples used in Figures 2 and 3 were used. b, d Immunoblot analysis of p62, NRF2 (b), and autophagy-related proteins (d). The same samples used in Figures 2 and 3 were used. Whole liver homogenates (45 $\mu$ g protein/lane) were separated using $10 \%$ SDS-PAGE and the band of $\beta$-actin (ACTB) was used as a loading control. Band intensities were measured densitometrically, normalized to the loading control, and expressed as values relative to those of regularly fed control HCVcpTg mice. Results were obtained from at least two independent immunoblot experiments and representative blots are shown. Data are expressed as the mean \pm SEM. ${ }^{*} p<0.05,{ }^{* *} p<$ 0.01 , and ${ }^{* * *} p<0.001$ between regularly fed control (Con) versus $30 \%$ reduction in diet (DR) groups.

anti-oxidant enzyme, was elevated in the DR group (Fig. 3c), as revealed by immunoblot analysis (Fig. 3e). The mRNA encoding other anti-oxidant enzymes, such as NAD(P)H quinone dehydrogenase 1 (NQO1), heme oxygenase 1 (HMOX1), superoxide dismutase 2 (SOD2), and catalase (CAT), were similar between the groups (Fig. 3c). The mRNA levels of DNA damageinducible transcript 3 protein (CHOP, encoded by Ddit3) and heat shock protein 5 (HSPA5), well-established ER stress indicators, and CHOP protein levels were significantly decreased in the DR group (Fig. 3d, e). These results exhibit that DR significantly reduces oxidative and ER stress, leading to suppression of NF- $\mathrm{kB}$ signaling in steatotic livers.

\section{DR Enhances Autophagy and p62/Sequestosome 1 Degradation in HCVcpTg Mice}

p62/sequestosome 1 (hereafter p62, encoded by Sqstm1) is an ubiquitin-binding autophagy adaptor protein that plays a crucial role in accelerating HCC initiation and progression, and its overexpression stabilizes nuclear factor erythroid 2-related factor 2 (NRF2, encoded by Nfe2l2), a key inducer of detoxifying and oxidative stress-eliminating enzymes, protecting malignant cells from oxidative stress-induced cell death $[28,29]$. 


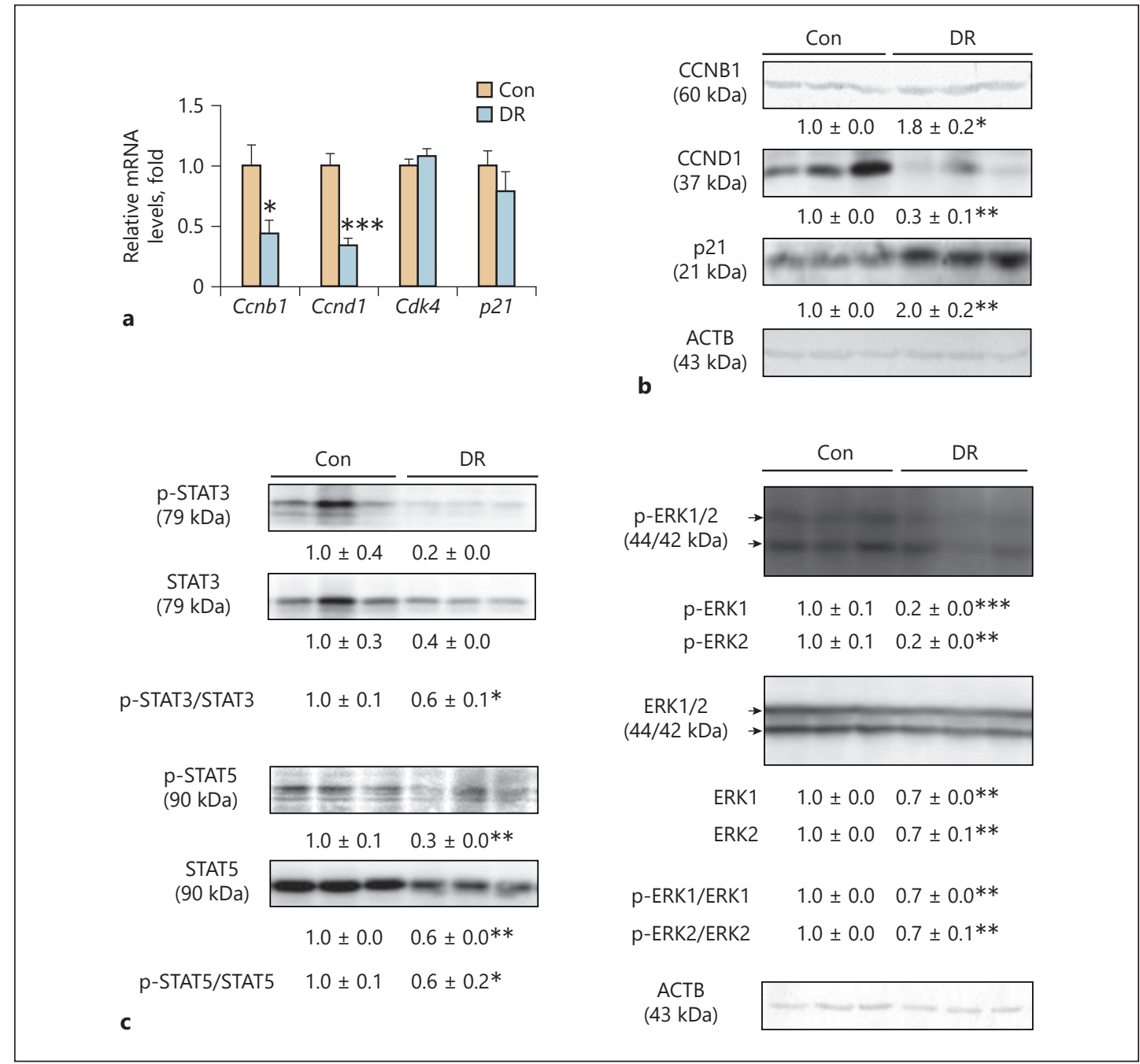

Fig. 5. 30\% DR attenuates cyclin D1 expression through suppressing STAT3, STAT5, and ERK signaling in HCVcpTg mice. a Hepatic mRNA levels of cell cycle regulators were quantified by qPCR, normalized to $18 \mathrm{~S}$ ribosomal RNA, and expressed as values relative to regularly fed control HCVcpTg mice. b, $\mathbf{c}$ Immunoblot analysis of cyclin B1, cyclin D1, p21 (b), and the total and phosphorylated form of STAT3, STAT5, and ERK1/2 (c). Whole liver homogenates (45 $\mu$ g protein/lane) were separated using $10 \%$ SDS-PAGE and the band of $\beta$-actin (ACTB) was used as a loading control. Band intensities were measured densitometrically, normalized to the loading control, and expressed as values relative to those of regularly fed control HCVcpTg mice. Results were obtained from at least two independent immunoblot experiments and representative blots are shown. d Immunohistochemical staining for p-STAT3 and p-ERK. p-STAT3 signals were intensely positive for hepatocyte nuclei in the control HCVcpTg mice, but such hepatocyte nuclei were not detected in the DR group. p-ERK signals were positive for hepatocytes and endothelial cells in the control HCVcpTg mice, and these signals were markedly attenuated in the DR group. The areas indicated by red boxes were enlarged and are shown in the respective lower row. Scale bar, $40 \mu \mathrm{m}$. Data are expressed as the mean \pm SEM. ${ }^{*} p<0.05$, ** $p<0.01$, and ${ }^{* * *} p<0.001$ between regularly fed control (Con) versus $30 \%$ reduction in diet (DR) groups.

(Figure continued on next page.)

Although mRNA levels of Sqstm1 and Nfe2l2 were similar between the groups (Fig. 4a), p62 protein levels were significantly lower in the DR group, while NRF2 showed no statistically significant difference between the groups (Fig. 4b). Since p62 protein was post-transcriptionally regulated by autophagy $[28,29]$, the expressions of key autophagy-associated molecules were assayed. The mRNA levels of microtubule-associated protein 1 light chain $3 \beta$ 


\section{Liver Cancer}

\begin{tabular}{|c|c|}
\hline Liver Cancer 2020;9:529-548 & \\
\hline DOI: $10.1159 / 000508308$ & $\begin{array}{l}\text { (c) } 2020 \text { The Author(s). Published by S. Karger AG, Basel } \\
\text { www.karger.com/lic }\end{array}$ \\
\hline
\end{tabular}

Jia et al.: Dietary Restriction Suppresses Steatosis-Derived Hepatocarcinogenesis

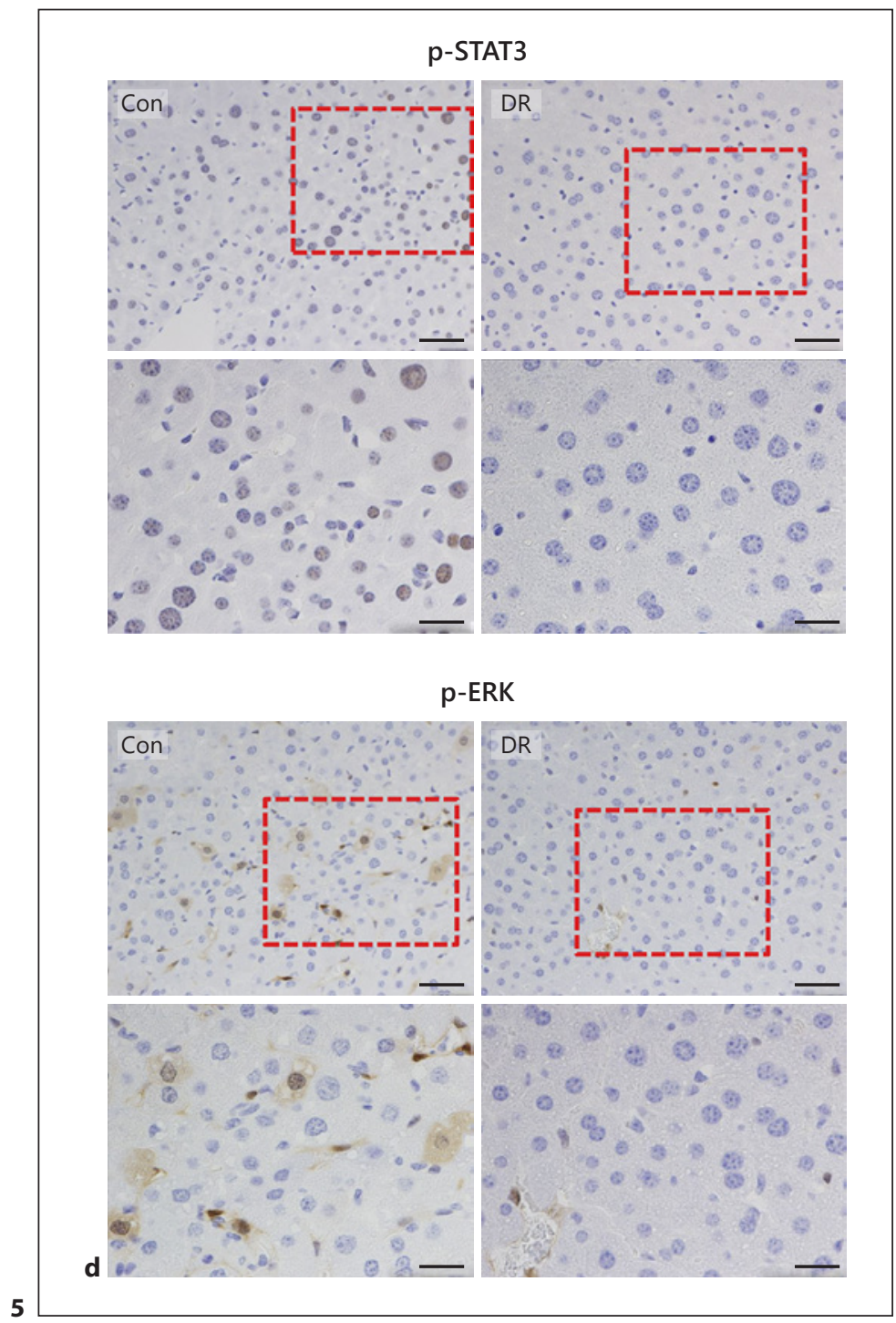

(LC3B, encoded by Map1lc3b), a classical autophagy substrate which is involved in autophagosome biogenesis, were elevated in the DR group (Fig. 4d). The mRNA levels of PTENinduced kinase 1 (PINK1), a key molecule of mitophagy, were simultaneously increased by DR, but did not reach statistical significance (Fig. 4c). Higher expression levels of essential proteins involved in the formation of autophagosomes, such as LC3, autophagy-related protein 5 (Atg5)-Atg12 complex were observed in the DR group (Fig. 4d). DR downregulates pro-tumorigenic p62 protein levels by enhancing autophagy, which may contribute to suppressed hepatic tumor development.

\section{DR Attenuates Growth Signaling in the Livers of HCVcpTg Mice}

Since disordered hepatocyte proliferation may cause the emergence of HCC, the expressions of cell cycle regulators were measured. Among conventional cyclins, cyclin-dependent kinases (CDKs), and CDK inhibitors, the mRNA levels of cyclin D1 (CCND1) and cyclin B1, encoded by Ccnd1 and Ccnb1, respectively, were significantly decreased by DR (Fig. 5a). The mRNA levels of cyclin A1, CDK1/2, and p53 were unaltered between the groups (online suppl. Fig. 1a). Immunoblot analysis revealed reduced CCND1 and increased p21 by DR (Fig. 5b). 


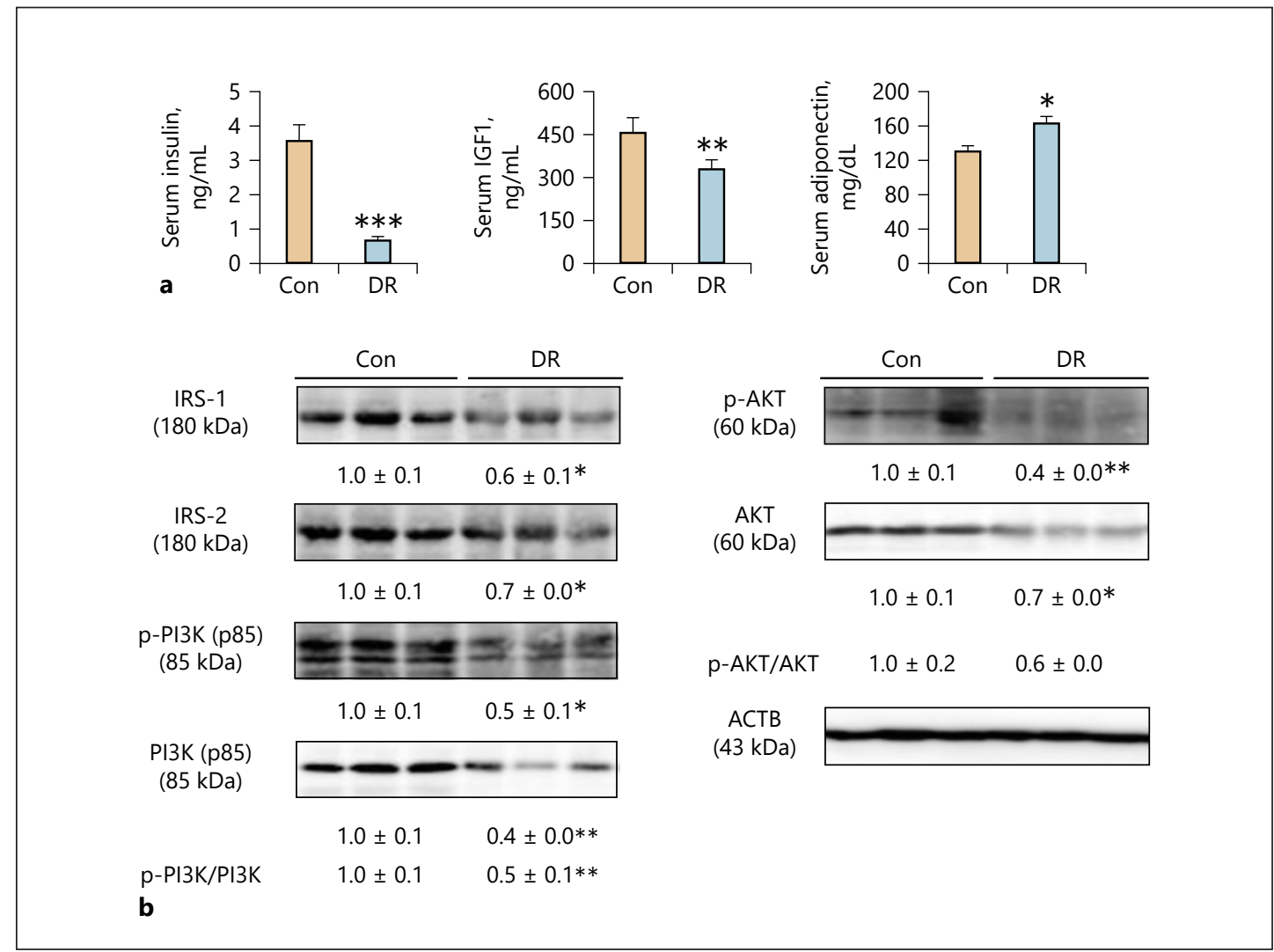

Fig. 6. 30\% DR downregulates hepatic insulin signaling in HCVcpTg mice. a Serum insulin, IGF1, and adiponectin levels. b Immunoblot analysis of IRS-1, IRS-2, and the total and phosphorylated form of PI3K and AKT. Whole liver homogenates ( $45 \mu \mathrm{g}$ protein/lane) were separated using 10\% SDS-PAGE and the band of $\beta$-actin (ACTB) was used as a loading control. Band intensities were measured densitometrically, normalized to the loading control, and expressed as values relative to those of regularly fed control HCVcpTg mice. Results were obtained from at least two independent immunoblot experiments and representative blots are shown. Data are expressed as the mean \pm SEM. ${ }^{*} p<0.05,{ }^{* *} p<0.01$, and ${ }^{* * *} p<0.001$ between regularly fed control (Con) versus $30 \%$ reduction in diet (DR) groups.

The Ccnd1 mRNA levels are reportedly regulated by NF- $\kappa$ B, STAT3, STAT5, and mitogen-activated protein kinase (MAPK) [30]. Indeed, DR significantly suppressed total STAT5 and extracellular signal-regulated kinase ERK1/2 protein levels, as well as phosphorylation of STAT3, STAT5, and ERK1/2 (Fig. 5c). DR did not exhibit any impact on phosphorylation of c-Jun $\mathrm{N}$-terminal kinase (online suppl. Fig. 1b). Immunostaining revealed that p-STAT3 signals were strongly positive in hepatocyte nuclei of the control HCVcpTg mice, but were absent in the DR group (Fig. 5d). p-ERK signals in hepatocytes and endothelial cells were blunted in the DR group (Fig. 5d). Collectively, DR downregulates CCND1 expression due to suppressing NF-кB, STAT3, STAT5, and ERK signaling pathways.

\section{DR Downregulates Hepatic Insulin Signaling in HCVcpTg Mice}

Insulin signaling is crucial not only for glucose homeostasis, but also for cell proliferation. After insulin and IGF1 bind to the insulin receptor, insulin receptor substrate (IRS)-1 and 2 transduce insulin signaling in the liver, resulting in activating downstream kinases such as phosphatidylinositol-3 kinase (PI3K) and serine/threonine-protein kinase AKT [31]. In the DR group, significant decreases in serum insulin and IGF1 and reciprocal increases in adiponectin, a key 


\begin{tabular}{|c|c|c|}
\hline \multirow[b]{2}{*}{ Liver Cancer } & \multicolumn{2}{|l|}{ Liver Cancer 2020;9:529-548 } \\
\hline & DOI: $10.1159 / 000508308$ & $\begin{array}{l}\text { O) } 2020 \text { The Author(s). Published by S. Karger AG, Basel } \\
\text { www.karger.com/lic }\end{array}$ \\
\hline
\end{tabular}

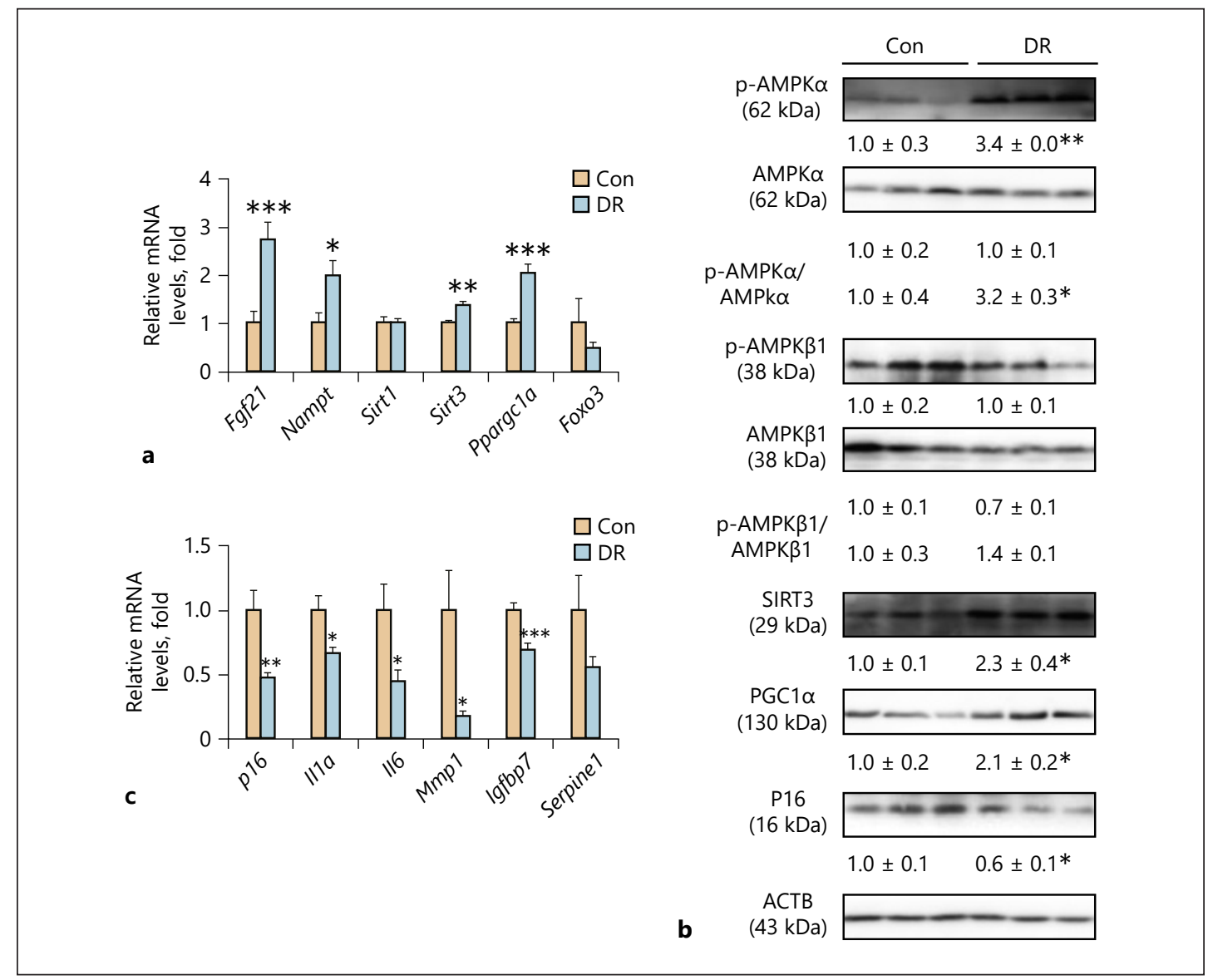

Fig. 7. 30\% DR moderates the hepatocyte senescence phenotype in HCVcpTg mice. a Hepatic mRNA levels of Fgf21, the NAD-surtuin pathway, and mitochondriogenesis were quantified by qPCR, normalized to $18 \mathrm{~S}$ ribosomal RNA, and expressed as values relative to regularly fed control HCVcpTg mice. b Immunoblot analysis of AMPK, SIRT3, PGC1 $\alpha$, and p16. Whole liver homogenates ( $45 \mu \mathrm{g}$ protein/lane) were separated using $10 \%$ SDS-PAGE and the band of $\beta$-actin (ACTB) was used as a loading control. Band intensities were measured densitometrically, normalized to the loading control, and expressed as values relative to those of regularly fed control HCVcpTg mice. Results were obtained from at least two independent immunoblot experiments and representative blots are shown. c Hepatic mRNA levels of SASP genes. The same assay was performed as in a. Data are expressed as the mean \pm SEM. ${ }^{*} p<0.05,{ }^{* *} p<0.01$, and ${ }^{* * *} p<0.001$ between regularly fed control (Con) versus $30 \%$ reduction in diet (DR) groups.

adipokine possessing insulin-sensitive and anti-cancer properties, were detected (Fig. 6a). In agreement with these findings, hepatic expression of IRS-1/2 and its downstream kinases, PI3K and AKT, was significantly reduced by $30 \%$ DR (Fig. 6b). These results indicate that DR suppresses hepatic insulin signaling.

\section{DR Decreases Hepatocyte Senescence Phenotype in HCVcpTg Mice}

Hepatocarcinogenesis is closely associated with senescence since age-dependent reductions in fatty catabolism causes lipotoxicity and oxidative stress, leading to accumulation of various abnormalities in hepatocytes [32]. Fibroblast growth factor 21 (FGF21, encoded by Fgf21) and AMP-activated protein kinase (AMPK) have significant roles in the regulation of energy balance, and there is convincing evidence that activating FGF21/AMPK can suppress hepatocarcinogenesis and extend the mammalian lifespan [33, 34]. The mRNA levels of Fgf21, 


\begin{tabular}{|c|c|c|}
\hline Rank & Gene & $\begin{array}{c}\text { Fold } \\
\text { (Con/DR) }\end{array}$ \\
\hline 1 & Tff3 & 787.6 \\
\hline 2 & Lcn2 & 87.6 \\
\hline 3 & Prtn3 & 76.3 \\
\hline 4 & Saa1 & 62.2 \\
\hline 5 & Saa2 & 52.1 \\
\hline 6 & Thrsp & 47.4 \\
\hline 7 & Sqle & 45.7 \\
\hline 8 & Sult7e1 & 43.9 \\
\hline 9 & Fasn & 43.5 \\
\hline 10 & Fabp5 & 34.9 \\
\hline
\end{tabular}
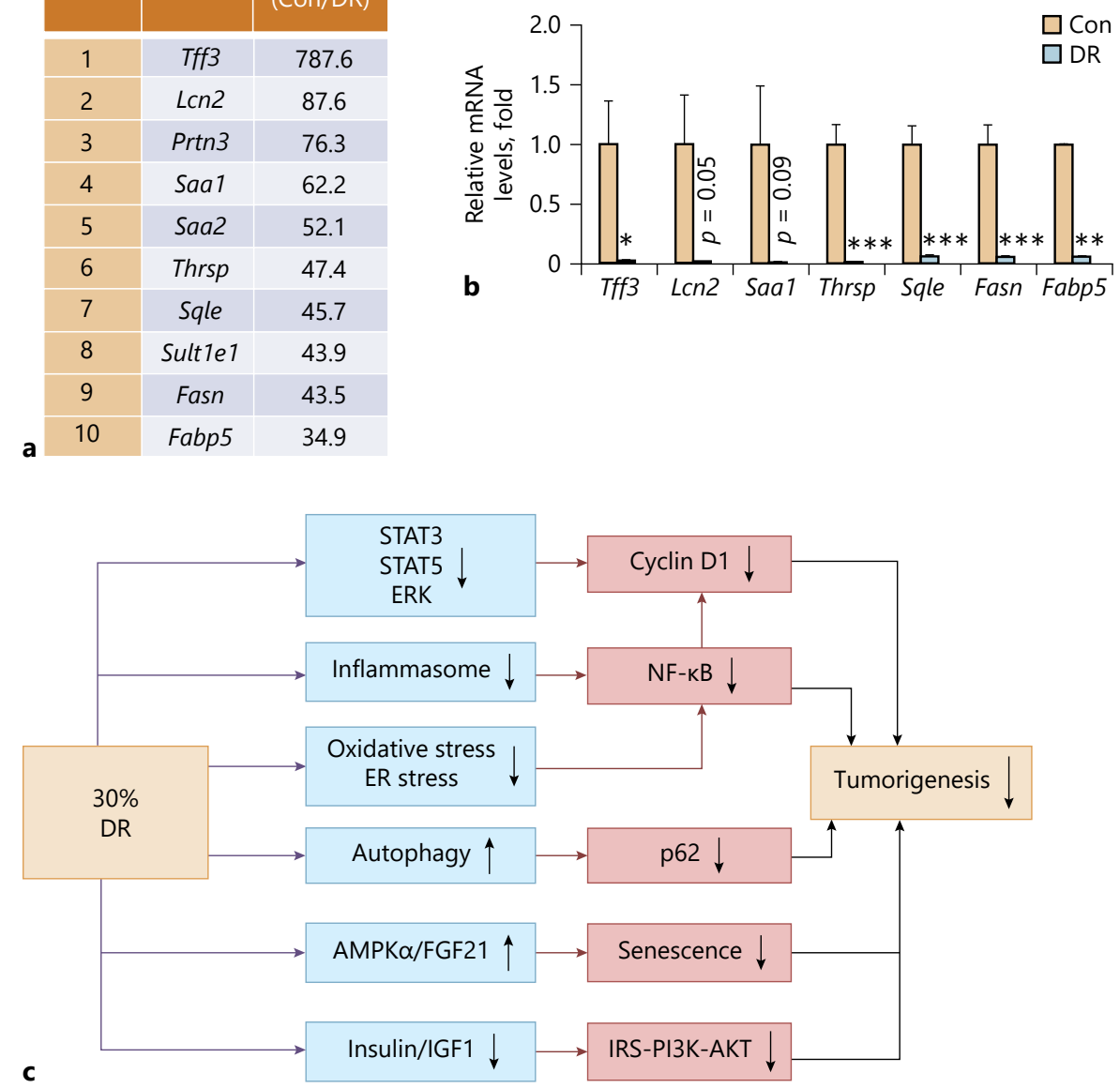

Fig. 8. STAT3 signaling and the de novo lipogenesis pathway are the most suppressed pathways by $30 \%$ DR in HCVcpTg mice. a The top 10 downregulated genes by DR, selected by microarray analysis. $\mathbf{b}$ Validation of microarray data shown in a by qPCR. The mRNA levels were normalized to $18 \mathrm{~S}$ ribosomal RNA, and expressed as values relative to regularly fed control HCVcpTg mice. c A proposed mechanism of how $30 \%$ DR prevents steatosis-induced hepatic tumorigenesis in HCVcpTg mice. Data are expressed as the mean \pm SEM. $* p<0.05$, ** $p<0.01$, and ${ }^{* * *} p<0.001$ between regularly fed control (Con) versus $30 \%$ reduction in diet (DR) groups.

nicotinamide phosphoribosyltransferase (NAMPT, encoded by Nampt), sirtuin 3 (SIRT3, encoded by Sirt3), and peroxisome proliferator-activated receptor- $\gamma$ coactivator- $1 \alpha$ (PGC1 $\alpha$, encoded by Ppargc1a) significantly raised by DR, while those of SIRT1 (Sirt1) and forkhead box 03 (Foxo3) remained unchanged (Fig. 7a). Immunoblot analysis uncovered marked increases in protein levels of SIRT3, PGC1 $\alpha$, and phosphorylated AMPK $\alpha$. No changes were detected in the expression of AMPK $\beta 1$ and its phosphorylated form (Fig. 7b).

Aging cells secrete several growth factors, cytokines, and proteases designated as senescence-associated secretory phenotype (SASP), driving oncogenesis. The qPCR analysis of SASP-related gene mRNAs revealed that most of the SASP mRNAs, including CDK inhibitor 2A (also known as $p 16$ ), Il1a, Il6, matrix metallopeptidase 13 (Mmp1), and IGF-binding protein 7 (Igfbp7) were decreased after DR (Fig. 7c). DR-induced decreases in a conventional senescence marker p16 were corroborated by immunoblot analysis (Fig. 7b). These results imply that DR modulates liver senescence, resulting in its anti-aging and anti-tumor properties. 
Jia et al.: Dietary Restriction Suppresses Steatosis-Derived Hepatocarcinogenesis

STAT3 Signaling and de novo Lipogenesis Are the Most Suppressed Pathway by DR

The abovementioned targeted analyses revealed marked downregulation of several key pro-oncogenic pathways due to chronic $30 \% \mathrm{DR}$, while it remained unclear which pathways were the most strongly altered. To further explore the mechanism by which DR suppressed liver tumorigenesis in HCVcpTg mice, a transcriptomic analysis was conducted. The top 10 gene mRNAs markedly downregulated by DR are listed in Fig. 8a. Among them, trefoil factor 3 (Tff3), lipocalin 2 (Lcn2), serum amyloid A1 (Saa1), thyroid hormone-responsive Spot14 (Thrsp), squalene epoxidase (Sqle), FA synthase (Fasn), and FA-binding protein 5 (Fabp5) mRNAs were downregulated as revealed by qPCR analysis (Fig. 8b). Interestingly, three of the top four gene mRNAs, Tff3, Lcn2, and Saa1, were previously reported as typical STAT3 target genes [35].

Among the remaining top 10 downregulated gene mRNAs, Thrsp, Sqle, and Fasn are involved in de novo FA/cholesterol synthesis. Indeed, the mRNA levels of lipogenic enzymes, such as Fasn, acetyl-CoA carboxylase- $\alpha$ (Acaca), and stearoyl-CoA desaturase 1 (Scd1) were significantly decreased in the DR group (online suppl. Fig. 2a). Additionally, the mRNA levels of enzymes involved in de novo cholesterogenesis, encoding 3-hydroxy-3-methylglutarylCoA synthase 1 (Hmgcs1) and reductase (Hmgcr), squalene synthase (farnesyl diphosphate farnesyl transferase 1, Fdft1) and epoxidase (Sqle) were downregulated by DR (online suppl. Fig. 2a). Therefore, STAT3 signaling and de novo lipogenesis were considered as the most suppressed pathway by continuous 30\% DR.

\section{DR Enhances FA Mitochondrial $\beta$-Oxidation without Peroxisome Proliferation and Increased Nuclear PPAR $\alpha$}

The changes in hepatic FA metabolism were also assessed. The mRNA levels of $C d 36$ and long-chain acyl-CoA synthetase 1 (Acsl1), involved in FA uptake from the blood and its conversion to acyl-CoA, respectively, were increased in the DR group (online suppl. Fig. 2a). The mRNA levels of genes encoding diacylglycerol 0-acyltransferase 1 (Dgat1) and apolipoprotein B (Apob), involved in TG incorporation and secretion from hepatocytes, respectively, were also enhanced by DR (online suppl. Fig. 2a, b). The mRNA levels of genes involved in mitochondrial FA $\beta$-oxidation, such as medium-chain and long-chain acyl-CoA dehydrogenase (MCAD and LCAD, encoded by Acadm and Acadl, respectively) were significantly increased by DR, while no remarkable change was observed in the mRNA levels of acyl-CoA oxidase 1 (ACOX1, encoded by Acox1), a rate-limiting enzyme of peroxisomal FA $\beta$-oxidation. Immunoblot analysis revealed increased protein levels of MCAD and LCAD, but neither ACOX1 nor peroxisomal 3-ketoacyl-CoA thiolase (PT). These results suggest suppression of lipogenesis and enhancement of FA uptake into hepatocytes, FA catabolism mainly in mitochondria, and TG secretion by DR.

The expression of FA $\beta$-oxidation enzymes, such as MCAD, LCAD, ACOX1, and PT, is induced by activation of nuclear receptor PPAR $\alpha$. PPAR $\alpha$ activation is also linked with peroxisome proliferation and hepatocarcinogenesis in rodents [14, 23]. However, DR did not increase the expression of peroxisomal enzymes ACOX1 and PT and 70-kDa peroxisomal membrane protein (PMP70), an indicator of peroxisome proliferation. Nuclear PPAR $\alpha$ protein levels were also similar between the groups (online suppl. Fig. 2c). DR-induced tumorsuppressing effect was likely independent of PPAR $\alpha$ activity.

DR Does Not Impact on Hepatic Fibrogenesis, Epithelial-to-Mesenchymal Transition, or Stemness in HCVcpTg Mice

Lastly, the impact of DR on hepatic fibrosis and epithelial-to-mesenchymal transition (EMT) was evaluated. The liver hydroxyproline contents and mRNA levels encoding transforming growth factor- $\beta 1$ (Tgfb1), connective tissue growth factor (Ctgf), $\alpha$-smooth muscle

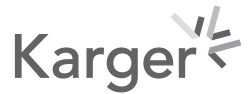


actin (Acta2), and collagen 1a1 (Col1a1) were comparable between the groups, which were consistent with the results of Azan-Mallory staining (online suppl. Fig. 3a, b, e). The mRNA levels of genes associated with stemness (Cd133, Cd44, and Aldh1a1) and EMT (Epcam, Vim, $C l d n 1$, and Cdh1) were similar between the groups, although $\alpha$-fetoprotein (Afp) was downregulated by DR (online suppl. Fig. 3c, d). Therefore, DR did not have a significant impact on hepatic fibrosis and stemness.

\section{Discussion}

The current study revealed that 30\% DR prevents steatosis-driven hepatic tumorigenesis in HCVcpTg mice. Mechanistically, DR attenuated oxidative and ER stress in the liver, suppressing the activation of inflammasome and NF- $\kappa$ B. DR also decreased CCND1 expression due to suppressing STAT3, STAT5, and ERK signaling, as well as NF- $\kappa$ B. Additionally, DR suppressed hepatic insulin/IGF1 signaling and reduced the expression of pro-tumorigenic molecules, such as p62, IRS, and SASP, and enhanced FGF21 and AMPK $\alpha$, which possess antiaging and anti-tumor properties. Based on the results obtained from the current study, we propose multiple beneficial effects of DR on preventing steatosis-associated hepatic tumorigenesis (Fig. 8c).

NF- $\kappa \mathrm{B}$ is a key transcription factor that has a critical role in the hepatocyte injury-inflammation-regeneration response leading to liver tumorigenesis [25-28]. Since the persistence of hepatic steatosis induces oxidative and ER stress and is regarded as a chronic low-grade hepatic inflammation state, it is reasonable to consider that hepatic microinflammation and persistent NF- $\kappa \mathrm{B}$ activation are crucial for steatosis-associated liver tumorigenesis. DR is known to improve the systemic inflammatory response [36], but the precise mechanism is not fully understood. A possible explanation for how DR efficiently downregulates hepatic $\mathrm{NF}-\kappa \mathrm{B}$ is that DR reduces hepatic lipotoxicity by inducing the anti-oxidant enzyme SOD1. SIRT3 was also reported to modulate the glutathione antioxidant defense system [37], eventually decreasing lipid peroxides and suppressing inflammasome/NF- $\mathrm{KB}$ activation. Additionally, DR increases serum adiponectin, hepatic AMPK, and FGF21, counteracting NF- $\kappa B$. A recent study revealed that short-term fasting reduced the number of circulating inflammatory monocytes and monocyte pro-inflammatory activity through activating hepatic AMPK and suppressing CCL2 production [38], and similar changes in AMPK and CCL2 were also found by long-term 30\% DR in the current study. Indeed, CCL2 was overexpressed in human liver cancers and was a prognostic factor for HCC patients [39]. Treatment of miR-122 knockout mice with a CCL2 inhibitor significantly reduced liver damage and HCC incidence [39]. Therefore, downregulating NF-kB-regulated pathways by DR may correct the hepatic steatoinflammatory microenvironment and prevent age-dependent steatosis-associated liver tumorigenesis.

STAT3 is another key contributor to hepatic inflammation, regeneration/proliferation, and tumorigenesis. Microarray analysis and immunostaining revealed that long-term 30\% DR markedly suppressed STAT3 signaling in hepatocytes, which may lower liver tumor occurrence in HCVcpTg mice. A close relationship between STAT3 activation and HCC has previously been documented. For example, hepatocyte-specific STAT3 disruption in mice resulted in more than a 6-fold reduction in HCC load induced by diethylnitrosamine (DEN), and knockdown of STAT3 led to death of DEN-induced HCC cells [40], suggesting that hepatocyte STAT3 plays an important role for HCC growth and HCC cell survival. TFF3 is one of the important downstream molecules of STAT3 [35]. Marked overexpression of TFF3 was reported in HCC tissues in mice and humans and the expression was strongly correlated with HCC aggressiveness, being high in poorly differentiated tumors [41]. A 2-year 30\% DR signif-

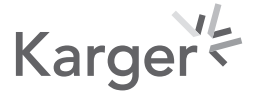


Jia et al.: Dietary Restriction Suppresses Steatosis-Derived Hepatocarcinogenesis

icantly upregulated microRNA-125a-5p, which acts as a tumor suppressor through downregulating STAT3 [42]. STAT3 is activated by cytokines, insulin and the other growth factors, and oxidative stress [43], and persistent 30\% DR drastically reduced these STAT3-activating factors and prevented liver tumorigenesis in the current study. Thus, a combination of STAT3/ $\mathrm{NF}-\kappa \mathrm{B}$ inhibition might be beneficial for preventing NAFLD- and metabolic syndrome-associated HCC, and DR might be a promising strategy for HCC prevention.

Another intriguing finding in this study was enhanced p62 degradation by autophagy. p62 is a recently proposed pro-oncogenic molecule and its accumulation is detected in not only HCC, but also the pre-malignant state of the liver, such as chronic hepatitis and NASH [28]. In fact, hepatocyte-specific p62-disrupted mice exhibited a 55\% lower HCC incidence induced by a high-fat diet and DEN compared with similarly treated mice [28]. Decreased p62 protein levels by autophagy contributes to attenuating liver tumor emergence and DR is thus a useful method to stimulate autophagy and p62 degradation.

The current study revealed that DR downregulated CCND1 expression. CCND1 is of vital importance in the cell cycle regulation and complex formation with CDK4/6 is required for G1/S transition. Like p62, CCND1 is often overexpressed in HCC and the surrounding tissues and regarded as one of the key driver genes for hepatocarcinogenesis [44]. Based on the results of this study, DR-induced suppression of NF- $\mathrm{BB}$, STAT3, STAT5, and ERK signaling is likely associated with downregulated CCND1 [30]. To our knowledge, this is the first demonstration that DR represses CCND1 expression. Further studies will be needed to clarify which nutrient restriction is mainly associated with downregulating cell cycle signaling.

STAT5 is activated through the stimulation of growth hormone and growth factors, as well as cytokines, and is translocated into nucleus to regulate its target genes, such as CCND1 and IGF1 [30, 45]. Since hepatocyte-specific Stat5 disruption in mice inhibited DEN-induced hepatocarcinogenesis [46], the downregulated STAT5 pathway may be responsible for suppressed liver tumorigenesis by DR.

IRS-1 is sometimes upregulated in human HCC [47]. Increases in total and phosphorylated AKT were observed in liver tumors and hepatocyte-specific disruption of Irs1, but not Irs2, exhibited suppression of DEN-induced hepatocarcinogenesis through lowering insulinmediated AKT phosphorylation in mice [48]. However, it was documented that adeno-associated virus-induced IRS-2 disruption repressed NAFLD-associated liver tumorigenesis in Pten/Sav1 double-knockout mice [49]. Taken together, lowering circulating insulin/IGF1, as well as attenuating hepatic IRS- 1 and 2 expression, would be a promising preventive strategy for HCC via downregulating the IRS-PI3K-AKT axis.

Previous studies demonstrated that DR delayed cancer progression in humans and experimental models by decreasing the rate of aging [50,51]. DR induced FGF21 and AMPK, protecting from aging-related cell dysfunction and exhibiting anti-inflammatory and antitumorigenic properties. Additionally, DR reduced hepatic expression of SASP, which secretes from senescent cells and promotes hepatocarcinogenesis. This result is partly consistent with a previous report showing that adult-onset, short-term DR reduced senescent cells in the liver and small intestinal epithelium, leading to improved mitochondrial function and reduced oxidative stress [52]. Since aging is a major risk factor for hepatocarcinogenesis, anti-senescence by DR might be beneficial to prevent hepatic tumorigenesis due to upregulating FGF21 and AMPK, as well as reducing SASP.

Since a potential beneficial effect of DR after chemotherapy was documented in patients with different types of cancer [53], the molecular mechanisms uncovered in this study may apply to humans, at least in part. However, there are some issues to be solved in further studies. First, can long-term DR prevent other types of hepatocarcinogenesis, i.e., chemically induced HCC or HCC developed from burned-out fibrotic NASH? Second, which is better for preventing steatosis-derived HCC, persistent uniform 30\% DR or intermittent DR? Also,

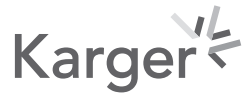


which nutrient or calorie restriction is responsible for HCC prevention, and how does DR alter microbiota and bacteria-producing metabolites? Lastly, will long-term administration with fasting mimics, such as metformin, exhibit similar beneficial effects to persistent DR? Clarifying these questions will shed new light on nutritional intervention to improve the outcome of steatosis-associated HCC.

\section{Acknowledgements}

We thank Dr. Takero Nakajima (Department of Metabolic Regulation, Shinshu University School of Medicine, Matsumoto, Japan), Ms. Ryoko Yada (Department of Gastroenterology, Kyushu Medical Center, Fukuoka, Japan), and DNA Chip Research Inc. (Tokyo, Japan) for technical support.

\section{Statement of Ethics}

All animal experiments were conducted in accordance with animal study protocols outlined in the Guide for the Care and Use of Laboratory Animals from the National Academy of Sciences and all protocols were approved by the Shinshu University School of Medicine animal care and use committee.

\section{Disclosure Statement}

The authors have no conflicts of interest to declare.

\section{Funding Sources}

No funding was acquired for this work.

\section{Author Contributions}

Conception and design: Fangping Jia, Naoki Tanaka, Toshifumi Aoyama. Acquisition, analysis, investigation, interpretation of data and statistical analysis: Fangping Jia, Pan Diao, Xiaojing Wang, Xiao Hu, Ibuki Nakamura, Saki Shirotori, Yoshiko Sato, Takefumi Kimura, Makoto Nakamuta, Naoki Tanaka. Supervision: Jun Nakayama, Kyoji Moriya, Kazuhiko Koike, Frank J. Gonzalez. Drafting the article: Fangping Jia, Naoki Tanaka. Critical revision of the manuscript for important intellectual content: all authors.

\section{References}

1 Saltiel AR, Olefsky JM. Inflammatory mechanisms linking obesity and metabolic disease. J Clin Invest. 2017 Jan; 127(1):1-4.

2 Blüher M. Obesity: global epidemiology and pathogenesis. Nat Rev Endocrinol. 2019 May;15(5):288-98.

3 Caldwell SH, Crespo DM, Kang HS, Al-Osaimi AM. Obesity and hepatocellular carcinoma. Gastroenterology. 2004 Nov; 127(5 Suppl 1):S97-103.

4 Dragani TA. Risk of HCC: genetic heterogeneity and complex genetics. J Hepatol. 2010 Feb;52(2):252-7.

5 Kimura T, Tanaka N, Fujimori N, Sugiura A, Yamazaki T, Joshita S, et al. Mild drinking habit is a risk factor for hepatocarcinogenesis in non-alcoholic fatty liver disease with advanced fibrosis. World J Gastroenterol. 2018 Apr;24(13):1440-50.

6 Kimura T, Kobayashi A, Tanaka N, Sano K, Komatsu M, Fujimori N, et al. Clinicopathological characteristics of non-B non-C hepatocellular carcinoma without past hepatitis B virus infection. Hepatol Res. 2017 Apr;47(5):405-18.

7 Tanaka N, Kimura T, Fujimori N, Nagaya T, Komatsu M, Tanaka E. Current status, problems, and perspectives of non-alcoholic fatty liver disease research. World J Gastroenterol. 2019 Jan;25(2):163-77.

8 Tanaka N, Aoyama T, Kimura S, Gonzalez FJ. Targeting nuclear receptors for the treatment of fatty liver disease. Pharmacol Ther. 2017 Nov;179:142-57. 


\begin{tabular}{|c|c|}
\hline Liver Cancer 2020;9:529-548 & \\
\hline DOI: $10.1159 / 000508308$ & $\begin{array}{l}\text { (c) } 2020 \text { The Author(s). Published by S. Karger AG, Basel } \\
\text { www.karger.com/lic }\end{array}$ \\
\hline
\end{tabular}

9 Anstee QM, Reeves HL, Kotsiliti E, Govaere O, Heikenwalder M. From NASH to HCC: current concepts and future challenges. Nat Rev Gastroenterol Hepatol. 2019 Jul;16(7):411-28.

10 O'Flanagan CH, Smith LA, McDonell SB, Hursting SD. When less may be more: calorie restriction and response to cancer therapy. BMC Med. 2017 May;15(1):106.

11 Nogueira LM, Lavigne JA, Chandramouli GV, Lui H, Barrett JC, Hursting SD. Dose-dependent effects of calorie restriction on gene expression, metabolism, and tumor progression are partially mediated by insulin-like growth factor-1. Cancer Med. 2012 Oct;1(2):275-88.

12 Moriya K, Yotsuyanagi H, Shintani Y, Fujie H, Ishibashi K, Matsuura Y, et al. Hepatitis C virus core protein induces hepatic steatosis in transgenic mice. J Gen Virol. 1997 Jul;78(Pt 7):1527-31.

13 Moriya K, Fujie H, Shintani Y, Yotsuyanagi H, Tsutsumi T, Ishibashi K, et al. The core protein of hepatitis C virus induces hepatocellular carcinoma in transgenic mice. Nat Med. 1998 Sep;4(9):1065-7.

14 Tanaka N, Moriya K, Kiyosawa K, Koike K, Aoyama T. Hepatitis C virus core protein induces spontaneous and persistent activation of peroxisome proliferator-activated receptor $\alpha$ in transgenic mice: implications for HCV-associated hepatocarcinogenesis. Int J Cancer. 2008 Jan;122(1):124-31.

15 Tanaka N, Moriya K, Kiyosawa K, Koike K, Gonzalez FJ, Aoyama T. PPARalpha activation is essential for HCV core protein-induced hepatic steatosis and hepatocellular carcinoma in mice. J Clin Invest. 2008 Feb;118(2):683-94.

16 Wang X, Tanaka N, Hu X, Kimura T, Lu Y, Jia F, et al. A high-cholesterol diet promotes steatohepatitis and liver tumorigenesis in HCV core gene transgenic mice. Arch Toxicol. 2019 Jun;93(6):1713-25.

17 Hu X, Wang X, Jia F, Tanaka N, Kimura T, Nakajima T, et al. A trans-fatty acid-rich diet promotes liver tumorigenesis in HCV core gene transgenic mice. Carcinogenesis. 2020;41(2):159-70.

18 Hu X, Tanaka N, Guo R, Lu Y, Nakajima T, Gonzalez FJ, et al. PPAR $\alpha$ protects against trans-fatty-acid-containing diet-induced steatohepatitis. J Nutr Biochem. 2017 Jan;39:77-85.

19 Tanaka N, Matsubara T, Krausz KW, Patterson AD, Gonzalez FJ. Disruption of phospholipid and bile acid homeostasis in mice with nonalcoholic steatohepatitis. Hepatology. 2012 Jul;56(1):118-29.

20 Tanaka N, Takahashi S, Hu X, Lu Y, Fujimori N, Golla S, et al. Growth arrest and DNA damage-inducible $45 \alpha$ protects against nonalcoholic steatohepatitis induced by methionine- and choline-deficient diet. Biochim Biophys Acta Mol Basis Dis. 2017 Dec;1863(12):3170-82.

21 Tanaka N, Takahashi S, Zhang Y, Krausz KW, Smith PB, Patterson AD, et al. Role of fibroblast growth factor 21 in the early stage of NASH induced by methionine- and choline-deficient diet. Biochim Biophys Acta. 2015 Jul; 1852(7):1242-52.

22 Tanaka N, Takahashi S, Matsubara T, Jiang C, Sakamoto W, Chanturiya T, et al. Adipocyte-specific disruption of fat-specific protein 27 causes hepatosteatosis and insulin resistance in high-fat diet-fed mice. J Biol Chem. 2015 Jan;290(5):3092-105.

23 Aoyama T, Peters JM, Iritani N, Nakajima T, Furihata K, Hashimoto T, et al. Altered constitutive expression of fatty acid-metabolizing enzymes in mice lacking the peroxisome proliferator-activated receptor alpha (PPARalpha). J Biol Chem. 1998 Mar;273(10):5678-84.

24 Nagaya T, Tanaka N, Suzuki T, Sano K, Horiuchi A, Komatsu M, et al. Down-regulation of SREBP-1c is associated with the development of burned-out NASH. J Hepatol. 2010 Oct;53(4):724-31.

25 Afonina IS, Zhong Z, Karin M, Beyaert R. Limiting inflammation-the negative regulation of NF- $\kappa \mathrm{B}$ and the NLRP3 inflammasome. Nat Immunol. 2017 Jul;18(8):861-9.

26 Maurel M, Samali A, Chevet E. Endoplasmic reticulum stress: at the crossroads of inflammation and metabolism in hepatocellular carcinoma development. Cancer Cell. 2014 Sep;26(3):301-3.

27 Kay J, Thadhani E, Samson L, Engelward B. Inflammation-induced DNA damage, mutations and cancer. DNA Repair (Amst). 2019 Nov;83(Jul):102673.

28 Umemura A, He F, Taniguchi K, Nakagawa H, Yamachika S, Font-Burgada J, et al. p62, upregulated during preneoplasia, induces hepatocellular carcinogenesis by maintaining survival of stressed HCC-initiating cells. Cancer Cell. 2016 Jun;29(6):935-48.

29 Moscat J, Karin M, Diaz-Meco MT. p62 in cancer: signaling adaptor beyond autophagy. Cell. 2016 Oct;167(3):606-9.

30 Klein EA, Assoian RK. Transcriptional regulation of the cyclin D1 gene at a glance. J Cell Sci. 2008 Dec;121(Pt 23):3853-7.

31 Siddle K. Signalling by insulin and IGF receptors: supporting acts and new players. J Mol Endocrinol. 2011 Jun; 47(1):R1-10.

32 Aravinthan AD, Alexander GJ. Senescence in chronic liver disease: is the future in aging? J Hepatol. 2016 Oct; 65(4):825-34

33 Jiang X, Tan HY, Teng S, Chan YT, Wang D, Wang N. The Role of AMP-activated protein kinase as a potential target of treatment of hepatocellular carcinoma. Cancers. 2019 May;11(5):E647.

34 Singhal G, Kumar G, Chan S, Fisher FM, Ma Y, Vardeh HG, et al. Deficiency of fibroblast growth factor 21 (FGF21) promotes hepatocellular carcinoma (HCC) in mice on a long term obesogenic diet. Mol Metab. 2018 Jul;13: 56-66.

35 Inagaki-Ohara K, Mayuzumi H, Kato S, Minokoshi Y, Otsubo T, Kawamura YI, et al. Enhancement of leptin receptor signaling by SOCS3 deficiency induces development of gastric tumors in mice. Oncogene. 2014 Jan; 33(1):74-84.

36 Park S, Park NY, Valacchi G, Lim Y. Calorie restriction with a high-fat diet effectively attenuated inflammatory response and oxidative stress-related markers in obese tissues of the high diet fed rats. Mediators Inflamm. 2012;2012:984643. 
37 Someya S, Yu W, Hallows WC, Xu J, Vann JM, Leeuwenburgh C, et al. Sirt3 mediates reduction of oxidative damage and prevention of age-related hearing loss under caloric restriction. Cell. 2010 Nov; 143(5):802-12.

38 Jordan S, Tung N, Casanova-Acebes M, Chang C, Cantoni C, Zhang D, et al. Dietary intake regulates the circulating inflammatory monocyte pool. Cell. 2019 Aug;178(5):1102-14.e17.

39 Li X, Yao W, Yuan Y, Chen P, Li B, Li J, et al. Targeting of tumour-infiltrating macrophages via CCL2/CCR2 signalling as a therapeutic strategy against hepatocellular carcinoma. Gut. 2017 Jan;66(1):157-67.

40 He G, Yu GY, Temkin V, Ogata H, Kuntzen C, Sakurai T, et al. Hepatocyte IKKbeta/NF-kappaB inhibits tumor promotion and progression by preventing oxidative stress-driven STAT3 activation. Cancer Cell. 2010 Mar; 17(3):286-97.

41 Okada H, Kimura MT, Tan D, Fujiwara K, Igarashi J, Makuuchi M, et al. Frequent trefoil factor 3 (TFF3) overexpression and promoter hypomethylation in mouse and human hepatocellular carcinomas. Int J Oncol. 2005 Feb;26(2):369-77.

42 Makwana K, Patel SA, Velingkaar N, Ebron JS, Shukla GC, Kondratov RV. Aging and calorie restriction regulate the expression of miR-125a-5p and its target genes Stat3, Casp2 and Stard13. Aging. 2017 Jul;9(7):1825-43.

43 Xiong A, Yang Z, Shen Y, Zhou J, Shen Q. Transcription factor STAT3 as a novel molecular target for cancer prevention. Cancers. 2014 Apr;6(2):926-57.

44 Deane NG, Parker MA, Aramandla R, Diehl L, Lee WJ, Washington MK, et al. Hepatocellular carcinoma results from chronic cyclin D1 overexpression in transgenic mice. Cancer Res. 2001 Jul;61(14):5389-95.

45 Chia DJ. Minireview: mechanisms of growth hormone-mediated gene regulation. Mol Endocrinol. 2014 Jul; 28(7):1012-25.

46 Kaltenecker D, Themanns M, Mueller KM, Spirk K, Golob-Schwarzl N, Friedbichler K, et al. STAT5 deficiency in hepatocytes reduces diethylnitrosamine-induced liver tumorigenesis in mice. Cytokine. 2019 Dec;124: 154573.

47 Cantarini MC, de la Monte SM, Pang M, Tong M, D’Errico A, Trevisani F, et al. Aspartyl-asparagyl beta hydroxylase over-expression in human hepatoma is linked to activation of insulin-like growth factor and notch signaling mechanisms. Hepatology. 2006 Aug;44(2):446-57.

48 Sakurai Y, Kubota N, Takamoto I, Obata A, Iwamoto M, Hayashi T, et al. Role of insulin receptor substrates in the progression of hepatocellular carcinoma. Sci Rep. 2017 Jul;7(1):5387.

49 Jeong SH, Kim HB, Kim MC, Lee JM, Lee JH, Kim JH, et al. Hippo-mediated suppression of IRS2/AKT signaling prevents hepatic steatosis and liver cancer. J Clin Invest. 2018 Mar;128(3):1010-25.

50 Fontana L, Nehme J, Demaria M. Caloric restriction and cellular senescence. Mech Ageing Dev. 2018 Dec;176: 19-23.

51 Zeng S, Shen WH, Liu L. Senescence and cancer. Cancer Transl Med. 2018 May-Jun;4(3):70-4.

52 Wang C, Maddick M, Miwa S, Jurk D, Czapiewski R, Saretzki G, et al. Adult-onset, short-term dietary restriction reduces cell senescence in mice. Aging. 2010 Sep;2(9):555-66.

53 Kopeina GS, Senichkin VV, Zhivotovsky B. Caloric restriction - A promising anti-cancer approach: from molecular mechanisms to clinical trials. Biochim Biophys Acta Rev Cancer. 2017 Jan;1867(1):29-41. 